\title{
Level of HER2/neu gene amplification as a predictive factor of response to trastuzumab-based therapy in patients with HER2-positive metastatic breast cancer
}

\author{
Giuseppe Gullo • Daniela Bettio • Valter Torri • \\ Giovanna Masci • Piermario Salvini • Armando Santoro
}

Received: 5 April 2008 / Accepted: 16 June 2008 /Published online: 29 July 2008

(C) The Author(s) 2008

\begin{abstract}
Summary To explore the clinical significance of the level of HER2/neu gene amplification in a homogenous cohort of 33 patients with HER2-positive metastatic breast cancer $(\mathrm{MBC})$ and available tumor samples treated with a trastuzumab-based regimen, we retrospectively performed dual-color fluorescence in-situ hybridization test and correlated them for each patient with time-to-progression (TTP) and overall survival (OS). We obtained values of HER2/chromosome 17 centromere (CEP17) ratio ranging from 2.5 to 21 (median 7.2). At the Cox model there is indication that patients whose tumors have high-level HER2/CEP17 ratio have shorter TTP and OS than those with lower ratio, when treated with a trastuzumab-based regimen. Correlations do not reach the limits of statistical significance but no formal sample size calculation was performed due to the explorative nature of the study. If
\end{abstract}

All Authors declare that they do not have conflict of interests.

G. Gullo $(\bowtie) \cdot$ G. Masci $\cdot$ A. Santoro

Oncology and Hematology Unit, Istituto Clinico Humanitas IRCCS,

Via Manzoni 56,

Rozzano, (Milan), Italy

e-mail: giuseppe.gullo@humanitas.it

\section{Bettio}

Laboratory of Cytogenetics, Istituto Clinico Humanitas IRCCS, Rozzano, (Milan), Italy

V. Torri

Clinical Oncology Research Unit,

Istituto di Ricerche Farmacologiche "Mario Negri",

Milan, Italy

P. Salvini

Medical Oncology Unit, Cliniche Gavazzeni,

Bergamo, Italy confirmed in larger cohorts of patients, HER2/CEP17 ratio could represent a reliable and economical predictor of response to trastuzumab-based therapy in MBC.

Keywords Trastuzumab - Metastatic breast cancer - FISH . Predictive factor $\cdot$ Monoclonal antibody

\section{Introduction}

The HER2/neu or c-erbB2 proto-oncogene, located on chromosome 17q11.2-q21, encodes a growth-factor receptor (HER2) belonging to the class I (epidermal growth factor) family of tyrosine kinases. Overexpression of the HER2 receptor, amplification of the HER2/neu gene, or both, occur in about $20-30 \%$ of human breast cancers and correlate with poor prognosis, including shorter disease-free survival and overall survival (OS) [1, 2]. Accurate assessment of HER2 status by determination of HER2 protein overexpression or gene amplification has become an essential part of management of breast cancer since the development of trastuzumab (Roche, Basel, Switzerland), a humanized monoclonal antibody that selectively targets the extracellular domain of the HER2. Trastuzumab is worldwidely approved as monotherapy or in combination with chemotherapeutic agents paclitaxel and docetaxel and with aromatase inhibitors for the treatment of metastatic HER2positive breast cancer, it is also approved as adjuvant therapy for HER2-positive early breast cancer [3-5].

The immunohistochemical technique reveals HER2 protein overexpression on cell surface, and when used according to the FDA-approved scoring system, provides a semiquantitative estimate HER2 expression [6]. It has emerged as the method of choice for screening. Fluorescence in situ hybridization (FISH), which detects gene amplification, is 
often used as a confirmatory test, particularly in cases with weak positive (score $2+$ ) immunohistochemical results.

Two FDA-approved FISH assays are currently available. The Inform kit (Ventana Medical Systems, Tucson, AZ, USA) uses a cosmid probe for the HER2/neu locus and it is considered positive when the absolute number of gene copies is $>4$, but cannot distinguish between true gene amplification or the presence of more gene copies due to chromosome 17 polysomy. The PathVysion kit (AbbottVysis, Downers Grove, IL, USA) includes a second probe for the centromeric region of chromosome 17 (CEP17), so making it possible to identify true gene amplification. The PathVysion test is considered positive (HER2 amplified) when the ratio HER2/CEP17 is $\geq 2$, but this ratio value is not routinely given in the patient's report.

HER2/neu amplification, when present in the primary tumor, is maintained throughout the course of disease and is an important predictor of response to chemotherapy, hormonal therapy, and mainly to trastuzumab-based regimens [7-9]. Our experience is that the numerical values of a positive PathVysion ratio vary widely from 2.1 to over 20 . However the biological and clinical implications of this variation have not been investigated. To address this, we performed PathVysion FISH analyses of all patients with HER2-positive metastatic breast cancer treated at our Institute over a defined period with a regimen including trastuzumab.

\section{Patients and methods}

\section{Study design}

This study was designed as a retrospective exploration of the relationship between level of HER2/neu amplification, and time to progression (TTP), time to central nervous system (CNS) progression, and OS. Specific inclusion criteria were: trastuzumab-based therapy for HER2-positive metastatic breast cancer; no previous treatment with trastuzumab, availability of tissue samples from the primary tumor or a metastatic site, and adequate treatment and follow-up information. Tests were performed prospectively on tumor samples archived in the Pathology Department of our hospital.

Clinical information was retrieved retrospectively from hospital records and placed in an ad hoc database. In line with standard procedures, all patients received pretreatment assessment comprising full blood count, liver and renal function tests, electrolytes, and baseline computed tomography of the chest, abdomen and pelvis, or alternatively, chest X-ray and ultrasound examination of the abdomen and pelvis. In conformity with standard policy at both hospitals, all patients gave written informed consent to the treatment received. Tumor assessment was performed every $9 \pm 1$ weeks. Disease response was assessed according to World Health Organization criteria [10].

Fluorescence in-situ hybridization

The PathVysion kits (Abbott-Vysis) include probes to determine the number of copies of HER2/neu (spectrum orange) and the number of CEP17 (spectrum green). Assays were performed on $3 \mu \mathrm{m}$-thick paraffin-embedded tissue sections, according to the manufacturer's instruction with the following minor modifications. Slides were aged three days at $50^{\circ} \mathrm{C}$ and dewaxed by washing in Microclearing (DiaPath, Bergamo, Italy) for 10 minutes twice, followed by incubation in 1:1 Microclearing/ethanol solution for 10 minutes. The waxed sections were dehydrated in $100 \%$ ethanol for 10 minutes, protease (Proteinase K, Sigma-Aldrich, Saint Louis, MO, USA) digested for 35 minutes at $50^{\circ} \mathrm{C}$ and washed for 3 minutes twice in Phosphate-Buffered Saline (PBS). Co-denaturation and hybridization then followed in a HYBrite oven (Abbott-Vysis) according to the manufacturer's protocol.

The FISH determinations were carried out in adherence to international guidelines by two cytogenetistics experienced in FISH techniques [11]. An Olympus BX 61 fluorescent microscope (Olympus, Tokyo, Japan) equipped with a Chroma (Rockingham, VT, USA) filter set (DAPI/ spectrum green/spectrum orange/triple band pass) was used to identify fluorescent signals. Before reviewing the FISH assay, the areas of invasive tumor, were identified using a parallel section stained with hematoxylin and eosin. The number of HER2/neu genes and chromosome 17 signals was scored between 20 and 60 non-overlapping cells where possible, using at least three distinct tumor fields. If a total of at least 20 non-overlapping, intact tumor nuclei could not be identified in distinct areas of the section, the FISH test was repeated in other sections of the tumor. In cases where the ratio was close to 2.0 (between 1.8 and 2.2), more than 60 cells were counted if possible, or the case rescored by another qualified observer to ensure the accuracy of the test results. The mean HER2 to chromosome 17 copy ratio was calculated. Tumors with a HER2/ CEP 17 ratio of more than 2 were considered positive for HER2/neu amplification.

Statistical analysis

TTP was calculated as the time from initiation of trastuzumab until disease progression or death without progression, which ever occurred first. Time to CNS progression was the time from initiation of trastuzumab until appearance of CNS disease, irrespective of whatever CNS was the first site of failure. OS was the time from 


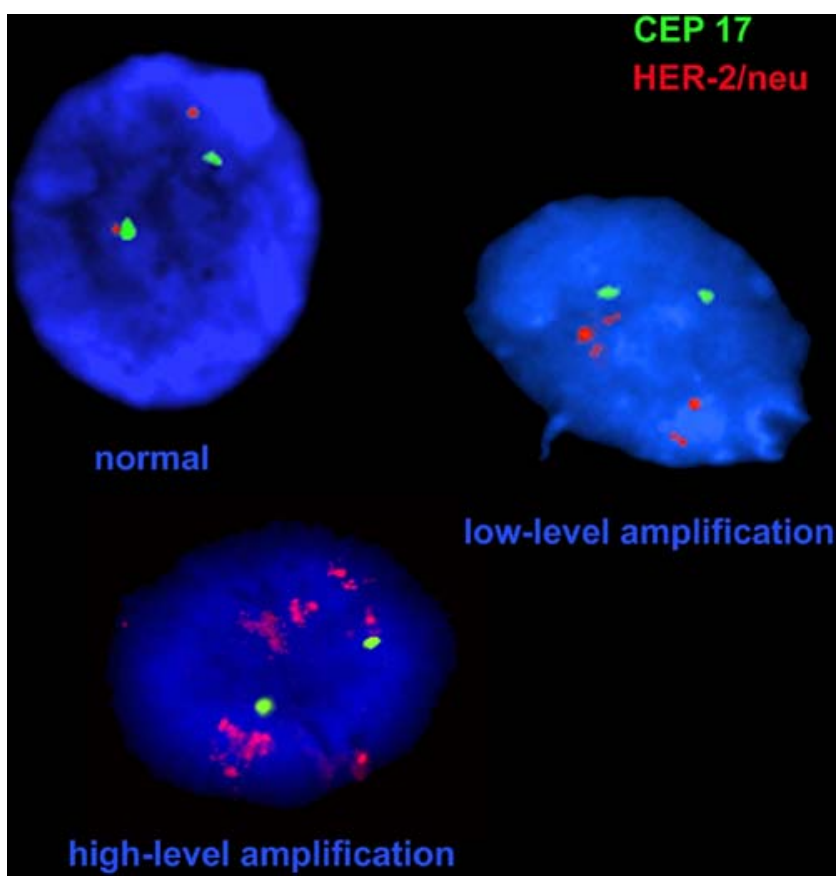

Fig. 1 A picture showing the differences in level of HER2/neu gene amplification in tumor specimens as evaluated by dual-color FISH test

initiation of trastuzumab until death for any cause. Surviving patients were censored on the day of the last contact. Time to event distributions were estimated using Kaplan-Meier curves and compared using the log-rank test. Contingency tables were used to describe distribution of molecular characteristics and established patient and tumor characteristics. The Cox proportional hazards models were used to assess the strength of the association of TTP, time to CNS progression, and OS and with various clinical and histological variables. Analysis was explorative in nature and no formal sample size calculation was performed.

\section{Results}

Thirty-five women with HER2-positive metastatic breast cancer received trastuzumab at Istituto Clinico Humanitas or Cliniche Gavazzeni between October 2001 and June 2006, and had tumor specimens available for analysis. Two of these women, both with a $3+$ score for HER2 by immunohistochemistry were excluded as no evidence of HER2 amplification was found by FISH. The remaining 33 women form the subject of the study. HER2/CEP17 ratio was assessed for all tumor samples with values ranging from 2.5 to 21 (median 7.2). The differences in level of HER2/neu amplification in tumor specimens as evaluated by FISH test can be observed in a picture at fluorescent microscope in Fig. 1.

The characteristics of the 33 patients are summarized in Table 1 together with the HER2/CEP17 ratios. Median age was 60 years (range: 27-73). Estrogen (ER) and/or progesterone receptors $(\mathrm{PgR})$ were positive in about $40 \%$. Trastuzumab was given in combination with chemotherapy in $27(82 \%)$ patients; whereas $6(18 \%)$ patients received trastuzumab alone. No patient had received trastuzumab previously.

Median TTP and OS for all patients were 10.3 and 20.3 months, respectively. CNS progression during trastuzumab therapy occurred in 7 patients $(21 \%)$. Figure 2 presents Kaplan-Meier TTP curves for the three subgroups defined by HER2/CEP17 ratio $(<6,6-10,>10.1)$. Chosen cut-offs correspond approximately with sample tertiles and are presented for displaying the association between level of HER2/neu amplification and TTP.

At the Cox model, there is indication that the risk of early progression during a trastuzumab-based therapy increases with increasing HER2/CEP17 ratio and that cases with high-level HER2/CEP17 ratio have a shorter TTP that those with lower ratio $(\mathrm{HR}=1.07 ; 95 \% \mathrm{CI}=0.98-1.16$; $p=$ 0.154). Cox analysis of HER $2 / \mathrm{CEP} 17$ ratio suggests an association with $\mathrm{OS}(\mathrm{HR}=1.0895 \% \mathrm{CI}=0.98-1.18, p=$ $0.124)$ but not with time to CNS progression ( $\mathrm{HR}=1.03$ $95 \% \mathrm{CI}=0.91-1.16 ; p=0.645)$.

Table 1 Patients characteristics and HER2/CEP17 ratio values obtained by dual-color FISH test

\begin{tabular}{lc}
\hline & Number $(\%)$ \\
\hline TOTAL & 33 \\
Median age (range) & $60(27-73)$ \\
Stage at diagnosis & \\
I & $2(6)$ \\
II & $10(30)$ \\
III & $15(46)$ \\
IV & $5(15)$ \\
Unknown & $1(3)$ \\
Histology & \\
Ductal & $31(94)$ \\
Other & $2(6)$ \\
Receptor status & \\
ER and PR negative & $19(58)$ \\
ER and/or PR positive & $14(42)$ \\
Grading & \\
G2 & $10(30)$ \\
G3 & $18(55)$ \\
Unknown & $5(15)$ \\
Visceral disease & $21(67)$ \\
Trastuzumab regimen & \\
Taxane & $22(67)$ \\
Vinorelbine & $5(15)$ \\
Trastuzumab alone & $6(18)$ \\
Median HER2/CEP17 ratio (range) & $7.2(2.5-21)$ \\
HER2/CEP17 ratio $<6$ & $13(39)$ \\
HER2/CEP17 ratio 6-10 & $11(33)$ \\
HER2/CEP17 ratio $>10.1$ & $9(28)$ \\
\hline
\end{tabular}


Fig. 2 Kaplan-Meier time to progression curves for the three subgroups defined by HER $2 /$ CEP17 ratio $(<6,6-10,>10.1)$. At the Cox model, there is indication that the risk of early progression during a trastuzumab-based therapy increases with increasing HER2/ CEP17 ratio $(\mathrm{HR}=1.07 ; 95 \%$ $\mathrm{CI}=0.98-1.16 ; p=0.154)$

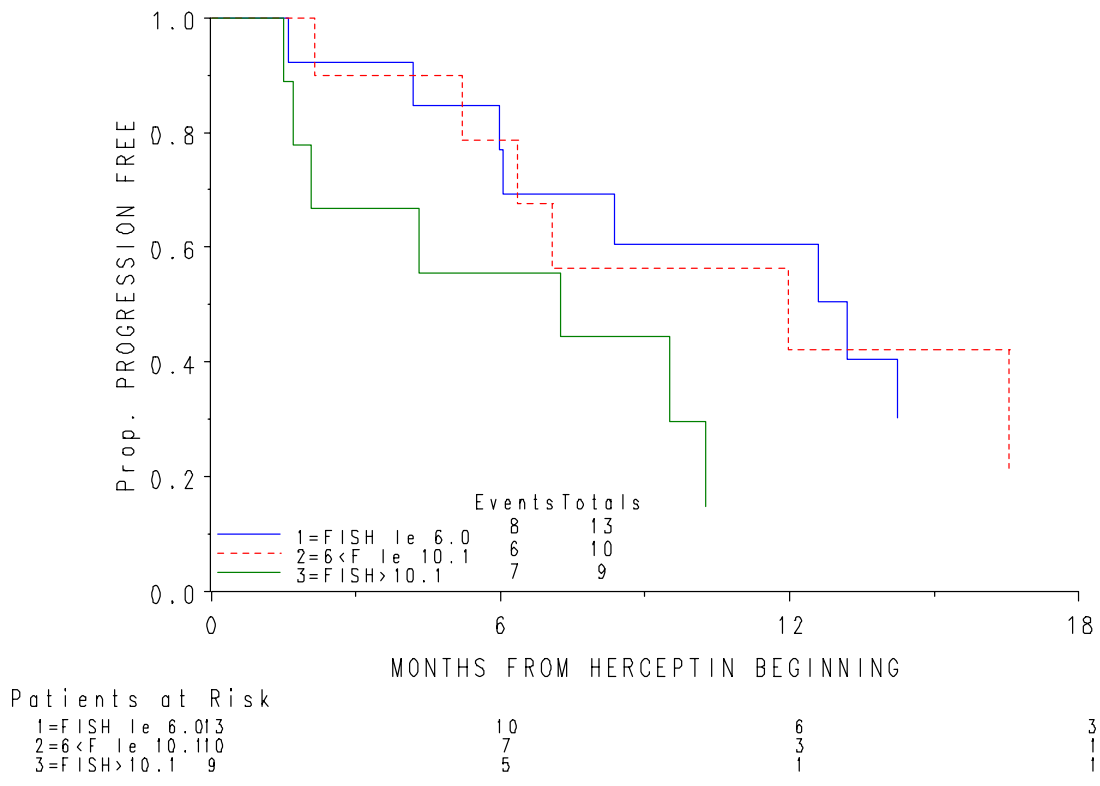

\section{Discussion}

Trastuzumab is now established in the treatment of HER2positive metastatic breast cancer [3-5]. Presence of HER2/ neu gene amplification, as determined by FISH, is significantly associated with response to trastuzumab treatment [9]. However, a number of patients with FISH-confirmed HER2positive disease fail to respond to trastuzumab, and show primary or acquired resistance to the antibody. In vitro and in vivo findings have suggested several reasons for trastuzumab resistance, involving the phosphorylation of HER2 tyrosine kinase [12], the signaling pathways associated with the HER family of receptors [13-16], and the truncated form of HER2 protein [17]. Very recently, a role for the antibody-dependent cell-mediated cytotoxicity of natural-killer cells/monocytes has been proposed to explain the differences in response to trastuzumab-based therapy in metastatic breast cancer [18]. So far, none of these potential predictors of response has been fully validated and routinely introduced in clinical practice.

In a previous study we found a significant association between tumor grade and level of HER2/neu amplification in a cohort of patients with early stage breast cancer [19]. In the present study we investigated HER2/neu amplification using a dual-color FISH test to determine the number of HER2/neu copies in relation to number of chromosome 17 copies in a small cohort of patients with HER2-positive metastatic breast cancer receiving trastuzumab. We found that TTP tended to be shorter in women with high HER2/ CEP17 ratio, although the difference was not statistically significant. Thus, increasing number of HER2/neu copies appears to augment the risk of disease progression during trastuzumab therapy. This finding, which requires confir- mation on a larger series of patients, suggests that cancers with a high number of HER2/neu copies may have different biological behavior compared to cancers with low gene copy number.

One explanation may be that a high level of amplification reflects a more complex genetic pattern and hence the overall dedifferentiation of a cancer. As might be expected, comparison of tumor samples representing different stages of tumor development, such as premalignant or in situ lesions, invasive cancers, and metastatic disease, has demonstrated that the overall number of copy increases during tumor progression. In addition to tumor progression, the high number of copy number aberrations has also been linked with poor patient prognosis [20]. Alternatively, high HER2/neu copy number may indicate activation of redundant trans-membrane signaling pathways that will not be adequately inhibited by trastuzumab. In both cases a trastuzumab-based therapy could not reach the same biological and clinical results observed in patients with a low-level HER2/neu amplification.

In conclusion, our is the first observation of a possible relation between level of HER2/neu amplification and TTP and OS. The most immediate practical implication of our finding is that the value of the HER2/CEP17 ratio should always be included in the FISH patient's report, not only to facilitate future studies in this area, but also to provide an indication of likely response to trastuzumab: metastatic breast cancer patients with high level of HER2/neu amplification might be at high risk of progression and should be closely monitored during trastuzumab-based therapy. Additional studies on larger cohorts of patients are required to further explore the prognostic implications of level of HER2/ neu amplification in metastatic breast cancer. 
Open Access This article is distributed under the terms of the Creative Commons Attribution Noncommercial License which permits any noncommercial use, distribution, and reproduction in any medium, provided the original author(s) and source are credited.

\section{References}

1. Slamon DJ, Clark GM, Wong SG et al (1987) Human breast cancer: correlation of relapse and survival with amplification of the Her-2/neu oncogene. Science 235:177-182

2. Ménard S, Casalini P, Tomasic G et al (1999) Pathobiologic identification of two distinct breast carcinoma subsets with diverging clinical behaviors. Breast Cancer Res Treat 55:169-177

3. Slamon DJ, Leyland-Jones B, Shak S et al (2001) Use of chemotherapy plus a monoclonal antibody against HER2 for metastatic breast cancer that overexpresses HER2. N Engl J Med 344:783-792

4. Vogel CL, Cobleigh MA, Tripathy D et al (2002) Efficacy and safety of trastuzumab as a single agent in first-line treatment of HER2-overexpressing metastatic breast cancer. J Clin Oncol 20:719-726

5. Marty M, Cognetti F, Maraninchi D et al (2005) Randomized phase II trial of the efficacy and safety of trastuzumab combined with docetaxel in patients with human epidermal growth factor receptor 2-positive metastatic breast cancer administered as firstline treatment: the M77001 study group. J Clin Oncol 23:42654274

6. Wolff AC, Hammond ME, Schwartz JN et al (2007) American Society of Clinical Oncology/College of American Pathologists guideline recommendations for human epidermal growth factor receptor 2 testing in breast cancer. J Clin Oncol 25:118-145

7. Pegram MD, Pauletti G, Slamon DJ (1998) HER-2/neu as a predictive marker of response to breast cancer therapy. Breast Cancer Res Treat 52:65-77

8. Lohrisch C, Piccart M (2001) HER2/neu as a predictive factor in breast cancer. Clin Breast Cancer 2:129-135
9. Mass RD, Press MF, Anderson S et al (2005) Evaluation of clinical outcomes according to HER2 detection by fluorescence in situ hybridization in women with metastatic breast cancer treated with trastuzumab. Clin Breast Cancer 6:240-246

10. Miller AB, Hoogstraten B, Staquet M et al (1981) Reporting results of cancer treatment. Cancer 47:207-214

11. Bilous M, Dowsett M, Hanna W et al (2003) Current perspectives on HER2 testing: a review of national testing guidelines. Mod Pathol 16:173-182

12. Giuliani R, Durbecq V, Di Leo A et al (2007) Phosphorylated HER-2 tyrosine kinase and Her-2/neu gene amplification as predictive factors of response to trastuzumab in patients with HER-2 overexpressing metastatic breast cancer (MBC). Eur J Cancer 43:725-735

13. Fujita T, Doihara H, Kawasaki K et al (2006) PTEN activity could be a predictive marker of trastuzumab efficacy in the treatment of ErbB2-overexpressing breast cancer. Br J Cancer 94:247-252

14. Harris LN, You F, Schnitt SJ et al (2007) Predictors of resistance to preoperative trastuzumab and vinorelbine for HER2-positive early breast cancer. Clin Cancer Res 13:1198-1207

15. Berns K, Horlings HM, Hennessy BT et al (2007) A functional genetic approach identifies the PI3K pathway as a major determinant of trastuzumab resistance in breast cancer. Cancer Cell 12:395-402

16. Valabrega G, Montemurro F, Aglietta M (2007) Trastuzumab: mechanism of action, resistance and future perspectives in HER2overexpressing breast cancer. Ann Oncol 18:977-984

17. Scaltriti M, Rojo F, Ocaña A et al (2007) Expression of p95HER2, a truncated form of the HER2 receptor, and response to anti-HER2 therapies in breast cancer. J Natl Cancer Inst 99:628-38

18. Musolino A, Naldi N, Bortesi B et al (2008) Immunoglobulin G fragment $\mathrm{C}$ receptor polymorphisms and clinical efficacy of trastuzumab-based therapy in patients with HER-2/neu-positive metastatic breast cancer. J Clin Oncol 26:1789-1796

19. Gullo G, Bettio D, Masci G et al (2006) The level of HER2/neu amplification may have a correlation with some relevant histopathological features of early stage breast cancer. Ann Oncol 17:s11

20. Kallioniemi A (2008) CGH microarrays and cancer. Curr Opin Biotechnol 19:36-40 